UDC $519.62 ; 530.145 ; 519.614$

DOI: $10.22363 / 2658-4670-2020-28-3-274-288$

\title{
Kuryshkin-Wodkiewicz quantum measurement model for alkaline metal atoms
}

\author{
Alexander V. Zorin \\ Peoples' Friendship University of Russia (RUDN University) \\ 6, Miklukho-Maklaya St., Moscow, 117198, Russian Federation
}

(received: June 10, 2020; accepted: September 14, 2020)

The constructive form of the Kuryshkin-Wodkiewicz model of quantum measurements was earlier developed in detail for the quantum Kepler problem. For more complex quantum objects, such a construction is unknown. At the same time, the standard (non-constructive) model of Holevo-Helstrom quantum measurements is suitable for any quantum object. In this work, the constructive model of quantum measurements is generalized to a wider class of quantum objects, i.e., the optical spectrum of atoms and ions with one valence electron. The analysis is based on experimental data on the energy ordering of electrons in an atom according to the Klechkovsky-Madelung rule and on the substantiation of a single-particle potential model for describing the energy spectrum of optical electrons in alkali metal atoms. A representation of the perturbation of a single-particle potential in the form of a convolution of the potential of an electron in a hydrogen atom with the Wigner function of a certain effective state of the core in an alkali metal atom representation allows reducing all calculation algorithms for alkali metals to the corresponding algorithms for the hydrogen atom.

Key words and phrases: models of quantum measurements, energy spectrum of alkali metal atoms, method of single-particle potential, perturbation of discrete spectrum of an observable

\section{Introduction}

The energy spectrum $E_{n}=-R /\left(2 n^{2}\right)$ of a valence electron in a hydrogen atom is described by the discrete spectrum of Hamiltonian $\hat{H}=-\Delta / 2-1 / r$ of the quantum Kepler problem with Hamilton function $H(q, p)=\vec{p}^{2} /(2 m)-$ $e^{2} /|\vec{r}|$. In addition to operator $\hat{H}$, the measured spectrum of the valence electron also depends on the state $\hat{\rho}$ of the quantum probe of a measuring instrument, i.e., is described by the discrete spectrum of the measured observable $O_{\rho}(H)=O_{W}\left(H * W_{\rho}\right)[1]$.

The constructive form of the Kuryshkin-Wodkiewicz quantum measurement model is thoroughly developed for the quantum Kepler problem [2] 
and quantum oscillator [3], [4]. For more complex quantum objects, such a construction is unknown. At the same time, the standard (nonconstructive) Holevo-Helstrom quantum measurement model is applicable to any quantum object, any quantum system [5], [6].

The goal of this paper is to generalize the constructive quantum measurement Kuryshkin-Wodkiewicz model to a wider class of quantum objects and to develop a quantum measurement model for optical spectrum of atoms and ions with one valence electron. We will consider the alkali metal atoms that consist of a core (atomic nucleus and electrons of all filled shells) and a valence (outer) electron, as well as ions with one valence electron.

\section{Hydrogen atom}

Quantum mechanics understands the description of the hydrogen atom as the description of the electron in this atom. Its energy spectrum has a very simple form

$$
E_{n}=-\frac{R}{2 n^{2}},
$$

when the atom is theoretically considered as an isolated quantum object. In the process of measurement, the quantum object is no more isolated, it becomes an open system incorporated in a more complex 'object + probe' quantum system [7]-[10].

The measured energy spectrum of an electron in a hydrogen atom is perturbed with respect to the spectrum (1): $\tilde{E}_{n}=E_{n}+\delta E_{n}$.

Thus, a problem of description (constructing a mathematical model) of the measured values of the hydrogen atom optical spectrum. This model incorporates the Weyl-Kuryshkin quantization rule and the rigging of the above mentioned model: $\left\{\varphi_{k}\right\}$ is the mixed state of the quantum probe, the smoothed (perturbed) classical observable is $A * W_{\left\{\varphi_{k}\right\}}(q, p)$, and the Weyl rule applied to it is $O_{\left\{\varphi_{k}\right\}}(A)=O_{W}(A)$. Theoretical study of the spectrum of this operator and the numerical calculation of the discrete spectrum parts affiliated with $\left\{\varphi_{k}\right\}$ are published in Ref. [11]. For the hydrogen atom the model is verified with the relative accuracy of $\sim 10^{-16}$.

Before discussing the dependence of the perturbation $\delta E_{n}$ in the hydrogen atom induced by the action of the measuring instrument with a quantum probe in the state $\left\{\varphi_{k}\right\}$, we recall what is known about the discrete energy spectrum $E_{n l}^{Z}$ of the valence electron in an isolated alkali metal atom with the atomic number $Z$.

\section{Energy spectrum of a valence electron in alkali metal atoms}

Quantum mechanical description of the valence electron in a hydrogen atom is provided by the Schrödinger equation

$$
\hat{H}_{0} \psi(\vec{r})=\varepsilon \psi(\vec{r})
$$


with the operator $\hat{H}_{0}=O_{W}(H)$, where $O_{W}$ is the Weyl quantization rule, transforming the Hamiltonian Kepler function $H=\vec{p}^{2} / 2-1 /|\vec{r}|$ into Hamiltonian operator $\hat{H}_{0}=-\vec{\nabla}^{2} / 2-1 /|\vec{r}|$. Equation (2) for the eigenvalues (the energy spectrum of the electron in the hydrogen atom) and eigenfunctions (the discrete spectrum wave functions of the hydrogen atom) has exact solutions [12]:

$$
\varepsilon_{n}=-\frac{1}{2 n^{2}}, \quad \psi_{n l m}(\vec{r})=R_{n l}(r) Y_{l m}(\theta, \varphi),
$$

where $\vec{r}=(r, \theta, \varphi)$ are spherical coordinates and $n, l, m$ are the principal, orbital, and azimuthal quantum number, respectively.

While in the hydrogen atom and hydrogen-like ions, the spectral lines are ordered according to the law

$$
-\frac{1}{2 n^{2}}
$$

i.e., the energy spectrum is degenerate with respect to the orbital and azimuthal quantum numbers, in the energy spectrum of valence (optical) electrons in the alkali metal atoms the orbital degeneracy is removed (see Eq. (4)). The degeneracy with respect to azimuthal quantum number remains in any potential having a spherical symmetry.

In the book by V.N. Kondratyev [13] in Table 9 on page 181 the following data are presented for the optical spectra of alkali metals (in Hartree atomic units):

$$
\varepsilon_{n l}=-\frac{1}{2(n-\delta(l))^{2}},
$$

namely:

(3) $\mathrm{Li}$ :

$$
\begin{array}{lll}
e 20=-0.1982754792, & e 21=-0.1302870145, \\
e 30=-0.07465200225, & e 31=-0.05710578080, & e 32=-0.05562970375, \\
e 40=-0.03883874030, & e 41=-0.03190061096, & e 42=-0.03128127346, \\
e 50=-0.02375325800, & e 51=-0.02033207896, & e 52=-0.02001600960, \\
e 60=-0.01601242872, & e 61=-0.01408066718, & e 62=-0.03128127346 ;
\end{array}
$$

(11) Na:

$$
\begin{aligned}
& e 30=-0.1888838814, \quad e 31=-0.1115650818, \quad e 32=-0.05592778605, \\
& e 40=-0.07245191330, \quad e 41=-0.05146315410, \quad e 42=-0.03140683790, \\
& e 50=-0.03800798912, \quad e 51=-0.02949906632, \quad e 52=-0.02008024064, \\
& e 60=-0.02335452320, \quad e 61=-0.01909585773, \quad e 62=-0.03140683790 ;
\end{aligned}
$$

(19) K:

$$
\begin{aligned}
& e 40=-0.1595965400, \quad e 41=-0.1010881942, \quad e 42=-0.03366251684, \\
& e 50=-0.06516440980, \quad e 51=-0.04810386124, \quad e 52=-0.02122122550, \\
& e 60=-0.03517930894, \quad e 61=-0.02802348772, \quad e 62=-0.03366251684 ;
\end{aligned}
$$


(37) Rb:

$$
\begin{aligned}
& e 50=-0.1534672079, \quad e 51=-0.09542857065, \quad e 52=-0.03523536413 \\
& e 60=-0.06354834915, \quad e 61=-0.04622131078, \quad e 62=-0.06530578980
\end{aligned}
$$

(55) Cs:

$$
\begin{aligned}
& e 60=-0.1431368322, \quad e 61=-0.09046170025, \quad e 62=-0.2075805080, \\
& e 70=-0.06074477220, \quad e 71=-0.04452676553 .
\end{aligned}
$$

As a result of splitting in the spectrum (4), the ordering of the spectral (energy) levels with increasing energy changes in comparison with the ordering in the hydrogen atom (3). This new ordering obeys the so-called KlechkovskyMadelung rule [14]-[16]. The relation of this order with the pairs $(n, l)$ of the principal and orbital quantum number is phenomenologically described by formula (4) and is determined by the increase of the pair $(n+l, l): 1 \mathrm{~s}, 2 \mathrm{~s}, 2 \mathrm{p}$, 3s, 3p, 4s, 3d, 4p, 5s, 4d, 5p, 6s, 4f, 5d, 6p, 7s, 5f, 6d, 7p, 8s, 5g. Here the values $l=0,1,2,3, \ldots$ correspond to the indices $s, p, d, f, \ldots$.

At the phenomenological level, the consideration of optical spectra of alkali metal atoms leads to a necessity of using potential function in the form [13]:

$$
V(r)=-\frac{e^{2}}{r}\left(1+c_{1}\left(\frac{r_{a}}{r}\right)+c_{2}\left(\frac{r_{a}}{r}\right)^{2}+c_{3}\left(\frac{r_{a}}{r}\right)^{3}+\ldots\right),
$$

where $c_{1}, c_{2}, \ldots$ are constants determined by the distortion character of the Coulomb field of the nucleus, $r_{a}$ being the Bohr radius (for hydrogen atom).

\section{Pseudopotential method for description of multielectron atoms}

For atoms having more than one electron, even the simplest ones, the Schrödinger equation can be solved directly neither analytically, nor by numerical methods. For this reason, the study of spectra of multielectron atoms is based on an approximate model. The approximation is based on the idea of self-consistent field that implies independent motion of each electron in a certain average field created by other electrons and atomic nucleus. A set of orbitals used for constructing the wave function is referred to as atomic configuration [11]. The most adequate scheme based on single-electron orbitals and atomic configuration is the Hartree-Fock approximation.

In fact the meaning of configuration is to assign to the entire atom a set of approximate integrals of motion, specified by set of labels $\left\{n_{j}, l_{j}, Z_{j}\right\}$, where the subscript $j$ enumerates the filled orbitals, and $Z_{j}$ is the occupation number, i.e., the number of electrons at the $j$-th orbital. The number of exact integrals of motion is typically insufficient for complete classification. Therefore, approximate but well-preserved integrals of motion are of primary importance [11]. To find out which orbitals in an atom are filled, the Aufbau rule should be used, namely, for ground state the distribution of electrons over the orbitals should correspond to the minimal energy compatible with 
the Pauli principle. The result of applying the Aufbau rule directly depends on the ordering of energy levels in the single-electron effective potential $U_{a}(r)$.

Far from the nucleus, the electron is subjected to the attractive Coulomb potential of the atomic nucleus, shielded by all other electrons, so that

$$
U_{a}(r) \sim-\frac{1}{r}, \quad r \gg r_{a},
$$

where $r_{a}$ is the characteristic radius of the atom. Near the nucleus, the shielding effect vanishes and the electron is attracted by the Coulomb potential of a bare atomic nucleus

$$
U_{a}(r) \sim-\frac{Z}{r}, \quad r \ll r_{a} .
$$

Considering the outer (valence) electrons, it is possible to construct a model of effective potential $U_{a}(r)$ based on the approximate behavior (5).

If the deviation of the effective single-electron potential $U_{a}(r)$ from the dependence $-1 / r$ can be considered a small perturbation, then the spectrum remains ordered like in a hydrogen atom. However, when considering the periodic law this is not true, which is an evidence of strong deviations of the effective potential from the Coulomb potential, leading to essential changes in the spectrum. An overlap of groups of energy levels with different principal quantum numbers $\mathrm{n}$ appears together with a new type of ordering according to $(n+l, n)$.

The notion of $n$-shell, i.e., states with the same principal quantum number $n$ arises from the fact that for pure Coulomb potential in a hydrogen atom these states are energy degenerate. If the potential slightly differs from the Coulomb one, the degeneracy is removed, but the energy levels with the same $n$ remain densely grouped in the energy scale. In this case, the notion of a shell remains physically significant. Otherwise, if the deviation from Coulomb potential is large, a complete regrouping of energy levels occurs and the hydrogen-like shells loose physical meaning, becoming merely formal entities. On the contrary, the notion of 'subshell' labelled by a pair of quantum numbers $\{n, l\}$ always remains significant for atoms, since the energy levels are degenerate with respect to azimuthal quantum number $m$ in any spherically symmetric potential.

A valence electron in an atom of alkali metal allows approximate quantum mechanical description using a single-particle model:

$$
\left[-\frac{\hbar^{2}}{2 m} \vec{\nabla}^{2}+V_{\text {eff }}(\vec{r})\right] \psi(\vec{r})=\varepsilon_{n l} \psi(\vec{r}) .
$$

For the first-order approximation the effective single-particle potential in the Thomas-Fermi method can be expressed as

$$
U_{a}^{T F}(r)=\frac{Z}{r} \chi(k r), \quad k=\left(\frac{8 \sqrt{2}}{3 \pi}\right)^{2 / 3} Z^{1 / 3} .
$$

through a table-defined function $\chi(r)$. 
Since the middle of the last century, researchers have been looking for a theoretical justification for the fact that in a many-electron atom the single-particle potential has a form that provides the "Aufbau rule" - the Klechkovsky-Madelung rule. In a number of articles and then in the book [15], Klechkovsky constructed this justification, starting from the Hartree-Fock approximation. The pseudopotential is expressed through the tabular function $\chi$, which is approximated by the expression:

$$
\chi(x)=(1+a x)^{-2} .
$$

At the same time, a number of researchers concluded that the desired pseudopotential has the form:

$$
U_{a}^{Z}(r)=-\frac{Z}{r\left(1+\frac{r}{R}\right)^{2}}, \quad R=\frac{1}{a \sqrt{Z}}\left(\frac{3 \pi}{8 \sqrt{2}}\right)^{2 / 3} .
$$

Demkov and Ostrovsky [17], [18] substantiated the existence of such a singleparticle potential proceeding from the geometric-symmetry model of the hydrogen atom by V.A. Fock [19].

$$
U_{\mu}^{D O}(r)=-\frac{2 \nu}{r^{2} R^{2}}\left[\left(\frac{r}{R}\right)^{\mu}+\left(\frac{R}{r}\right)^{\mu}\right]^{-2} .
$$

In [17], [18] Demkov and Ostrovsky established that equation (7) provides the most precise description of Klechkovsky-Madelung rule at $\mu=1 / 2$. In subsequent papers [20], [21] Demkov and Ostrovsky's proof was questioned, but the result was confirmed.

Remark 1. Subsequently, when considering the multielectron atom in the framework of the quantum field theory, Kholodenko et al. [22], [23] confirmed the correctness of the Demkov-Ostrovsky proof, and also generalized this result to such a degree that he began to describe not only the KlechkovskyMadelung rule, but also exceptions to it (see also [24], [25]).

These potentials are in good agreement with the experimentally observed spectra:

$$
\varepsilon_{n l}=-\frac{1}{2(n+\delta(l))^{2}}=-\frac{1}{2 n_{\mathrm{eff}}^{2}} .
$$

in Hartree atomic units.

The solution obtained is not universal, since there are exceptions to the Madelung rule in transition metals, as well as among lanthanides and actinides. Quantum-mechanically, these exceptions, as well as the rule itself, are still considered using relativistic Hartree-Fock calculations. The results obtained do not yet detail the exceptions. Kholodenko and Kaufman [23], using quantum field theory, showed that the Demkov-Ostrovsky potential does indeed give the correct answer. In addition, thanks to work [26], it became possible to identify the Demkov-Ostrovsky potential $U_{1 / 2}^{D O}(r)$ with the HartreeFock potential. 
Remark 2. It was shown in [22] that, confining oneself to quantum mechanical methods, it is impossible to derive Madelung's rule with exceptions. Madelung's rule and its exceptions contain much more information than is required for its use in chemistry. Recall that the invention of quantum mechanics in 1925-1926 was initially driven by the needs of atomic physics. Subsequently, quantum mechanics was extended to quantum field theory, which led to the development of the Standard Model of particle physics. Not surprisingly, particle physics methods have recently been applied to the periodic table of elements [25], [27]. In [22], it was demonstrated that the Madelung rule and its exceptions can be described within the framework of this model.

\section{Modeling the measured energy spectrum of alkali metal atoms}

So far, we have mainly dealt with the study of the quantum-measured energy state of the valence electron in the hydrogen atom. The energy spectrum of a non-measured (isolated) hydrogen atom has the form $\varepsilon_{n l}=$ $-1 /\left(2 n^{2}\right)$, degenerate with respect to the orbital quantum number $l$. The measurement procedure slightly perturbs potential energy $V_{0}(\vec{r})=-1 / r$ of the isolated hydrogen atom to $V_{\rho}(\vec{r})=-1 / r+\delta V_{\rho}(\vec{r})$, so that the perturbed spectrum of the hydrogen atom subjected to measurement has the form $\varepsilon_{n l}^{\kappa}=-1 /\left(2 n^{2}\right)+\delta E_{\kappa}^{1}$. Under such weak perturbation, the ordering of spectral lines remains unchanged.

However, even in first spectral lines of alkali metals the KlechkovskyMadelung ordering is observed.

In "hydrogen-like" alkali metal atoms the spectral terms have the form

$$
E_{n l}^{Z}=-\frac{R}{2\left(n+\sigma_{l}^{Z}\right)^{2}} .
$$

In the process of measurement, the "measured" energy spectrum takes the form

$$
E_{n l, \kappa}^{Z}=-\frac{R}{2\left(n+\sigma_{l}^{Z}\right)^{2}}+\delta E_{\kappa}^{Z},
$$

where the contributions $\delta E_{\kappa}^{1}$ and $\delta E_{\kappa}^{Z}$ are obtained from convolution $V(r) *$ $W_{\left\{\varphi_{k}\right\}}(q, p)$, where

$$
\begin{gathered}
V^{1}(r)=-\frac{1}{r} \\
U_{a}^{Z}(r)=-\frac{Z}{r\left(1+\frac{r}{R}\right)^{2}}, \quad R=\frac{1}{a \sqrt{Z}}\left(\frac{3 \pi}{8 \sqrt{2}}\right)^{\frac{2}{3}} .
\end{gathered}
$$

Therefore, the Weyl-Kuryshkin quantization rule is adequate for alkali metals to the same degree as adequate is potential $V_{\kappa}(r, \vartheta)$ that takes into account small corrections to the centrally symmetric potential. 
Ref. [23] shows the coincidence of the Demkov-Ostrovsky potential (7) with the Hartree-Fock potential (6). Similar to the potential $V_{\rho}(\vec{r})$ from the Kuryshkin-Wodkiewicz quantization rule [28], potential $V^{Z}(\vec{r})$ from equation (8) is an $\hat{H}_{0}$-compact perturbation of the potential $V^{1}(\vec{r})$ that equals zero at infinity (see the papers by B. Simon [29], [30] about Kato theorems). Thus, potential $U_{\mu}^{D O}(r)$ satisfies the conditions of the theorem from Ref. [31].

\section{Kuryshkin-Wodkiewicz quantum measurement model for alkali metal atoms and ions with one valence electron}

For valence electron in a hydrogen atom the potential has the form $V_{1}(r)=-1 / r$ and the energy spectrum is $\varepsilon_{n}=-1 /\left(2 n^{2}\right)$. In the process of measurement with an instrument whose quantum part is in the state $\rho=\sum c_{j}\left|\psi_{j}\right\rangle\left\langle\psi_{j}\right|$ both the potential and the spectrum are perturbed:

$$
\begin{gathered}
\rho: V_{1}(r) \mapsto V_{\rho}(r)=V_{1}(r)+\delta V_{\rho}(r), \\
\rho: \varepsilon_{n}=-\frac{1}{2 n^{2}} \mapsto \varepsilon_{n l}^{\rho}=-\frac{1}{2 n^{2}}+\delta \varepsilon_{n l}^{\rho} .
\end{gathered}
$$

In this case $V_{\rho}(r)=\left(V_{1} * W_{\rho}\right)(r)$;

$$
\begin{aligned}
O_{\rho}(H)=O_{W}\left(H * W_{\rho}\right)= & O_{W}\left(\left(\frac{p^{2}}{2}-\frac{1}{r}\right) * W_{\rho}\right)= \\
& =-\frac{1}{2} \nabla^{2}+C_{\rho} \hat{I}+\left(V_{\rho}=V_{1} * W_{\rho}=V_{1}+\delta V_{\rho}\right) \hat{I} .
\end{aligned}
$$

For a valence electron in an atom of alkali metal, the potential has asymptotic expressions

$$
-\frac{Z}{r} \underset{r \rightarrow 0}{\longleftarrow} V^{Z}(r) \underset{r \rightarrow \infty}{\longrightarrow}-\frac{1}{r}
$$

approximately equals $V_{\text {eff }}(r)$ from the Hartree-Fock method and conventionally has the form

$$
U_{1 / 2}^{D-O}(r)=-\frac{2 \nu}{r^{2} R^{2}}\left[\sqrt{\frac{r}{R}}+\sqrt{\frac{R}{r}}\right]^{-2} .
$$

Then the spectrum is traditionally presented in the form

$$
\varepsilon_{n l}^{Z}=-\frac{1}{2\left(n_{\mathrm{eff}}^{Z}\right)_{n l}^{2}}=-\frac{1}{2\left(n+\sigma_{\mathrm{eff}}^{Z}\right)_{n l}^{2}} .
$$

According to the theorem of Ref. [31], there exists such an abstract state $\rho_{Z}=\sum f_{j}\left|\psi_{j}\right\rangle\left\langle\psi_{j}\right|$, that

$$
\rho_{Z}: V_{1}(r) \mapsto V_{Z}(r)=\left(V_{1} * W_{\rho Z}\right)(r)=V_{1}(r)+\delta V_{Z}(r),
$$




$$
\rho_{Z}: \varepsilon_{n}=-\frac{1}{2 n^{2}} \mapsto \varepsilon_{n l}^{Z}=-\frac{1}{2\left(n_{\mathrm{eff}}^{Z}\right)_{n l}^{2}}=-\frac{1}{2\left(n+\sigma_{\mathrm{eff}}^{Z}\right)_{n l}^{2}} .
$$

Now let us apply the procedure of measuring the energy spectrum of the valence electron in an alkali metal atom with an instrument whose quantum part is in the state $\rho_{a p}=\sum c_{j}\left|\psi_{j}\right\rangle\left\langle\psi_{j}\right|$. As a result of the measurement the perturbation of the pseudopotential and the spectrum will occur:

$$
\begin{gathered}
\rho_{a p}: V_{Z}(r) \mapsto V_{\rho_{a p}}^{Z}(r)=\left(V_{Z} * W_{\rho_{a p}}\right)(r)=V_{Z}(r)+\delta V_{\rho_{a p}}^{Z}(r), \\
\rho_{a p}: \varepsilon_{n l}^{Z}=-\frac{1}{2\left(n_{\mathrm{eff}}^{Z}\right)_{n l}^{2}} \mapsto \varepsilon_{n l}^{Z}+\left(\delta \varepsilon_{\rho_{a p}}^{Z}\right)_{n l} .
\end{gathered}
$$

Let us rewrite relation (13) in more detail

$$
\begin{aligned}
V_{\rho_{a p}}^{Z}(r) & =\left(V_{z} * W_{\rho_{a p}}\right)(r)=\left(\left(V_{1} * W_{\rho z}\right) * W_{\rho_{a p}}\right)(r)= \\
& =\left(\left(V_{1}+\delta V_{Z}\right) * W_{\rho_{a p}}\right)(r)=V_{1}(r)+\delta V_{\rho}(r)+\left(\delta V_{z} * W_{\rho_{a p}}\right)(r)
\end{aligned}
$$

In this case

$$
H^{Z} * W_{\rho_{a p}}=\left(H_{1} * W_{\rho_{Z}}\right) * W_{\rho_{a p}}=\left(\left(\frac{p^{2}}{2}-\frac{1}{r}\right) * W_{\rho_{Z}}\right) * W_{\rho_{a p}}
$$

and

$$
\begin{aligned}
O_{\rho}(H)=O_{W}(H * W \rho) & =O_{W}\left(\left(\frac{p^{2}}{2}-\frac{1}{r}\right) * W_{\rho}\right)= \\
& =-\frac{1}{2} \nabla^{2}+C_{\rho} \hat{I}+\left(V_{\rho}=V_{1} * W_{\rho}=V_{1}+\delta V_{\rho}\right) \hat{I}
\end{aligned}
$$

\section{Discussion}

Ref. [28] describes a program calculating elements of the Ritz matrix and storing them in external files. According to the Ritz method, the eigenvalues of the Ritz matrix are spectral values of the quantity under study, i.e., the energy. This algorithm consists in solving generalized eigenvalue problem $M \vec{x}=B \vec{x}$, where $M$ is the Ritz matrix and $B$ is the matrix of pair scalar products of auxiliary functions in the Kuryshkin quantum mechanics. The program allows calculating Ritz matrices of arbitrary dimension. However, there are hardware limitations. To date the calculations are possible for matrix dimension of 55 and 91 . Parameter $E_{0}$ is the only one to be fitted to experimental data. For example, Figures 1 and 2 show the dependences of discrepancies on $E_{0}$ for first few energy levels of lithium atom and Figure 3 for sodium atom. The discrepancy functions are seen to have expressed minima that determine the effective fitted values of the parameters. 


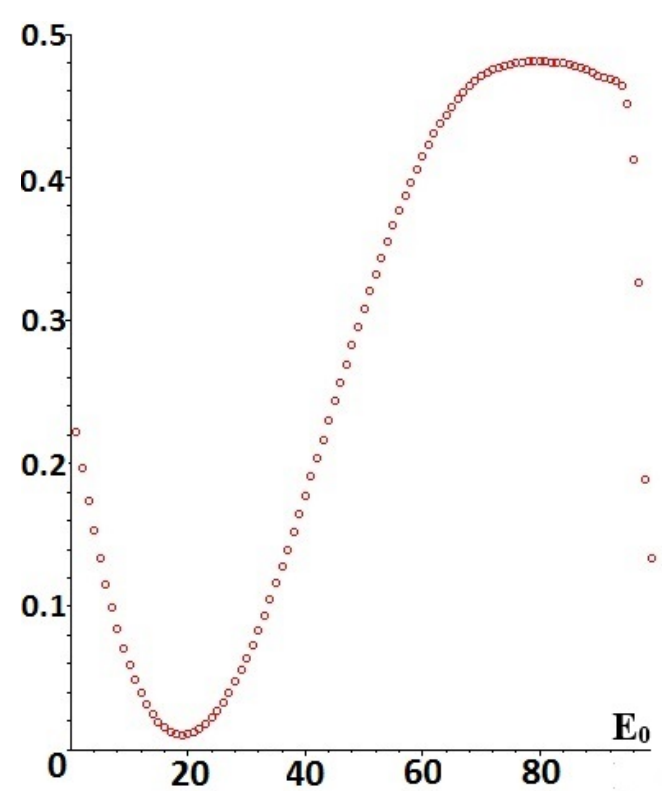

Figure 1. Quadratic discrepancy versus parameter $E_{0}$ for a group of first four energy levels of a lithium atom

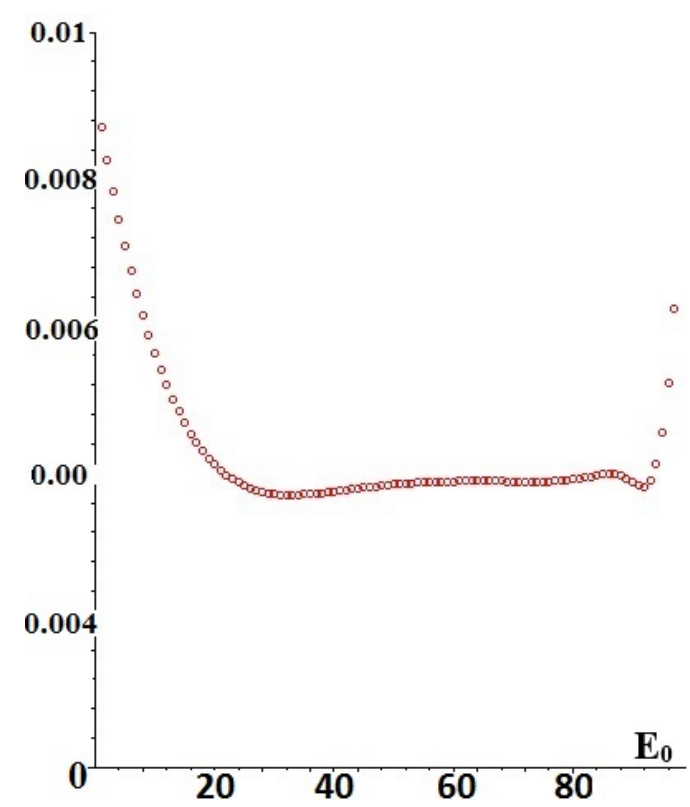

Figure 2. Quadratic discrepancy versus parameter $E_{0}$ for a group of first 8 energy levels of a lithium atom

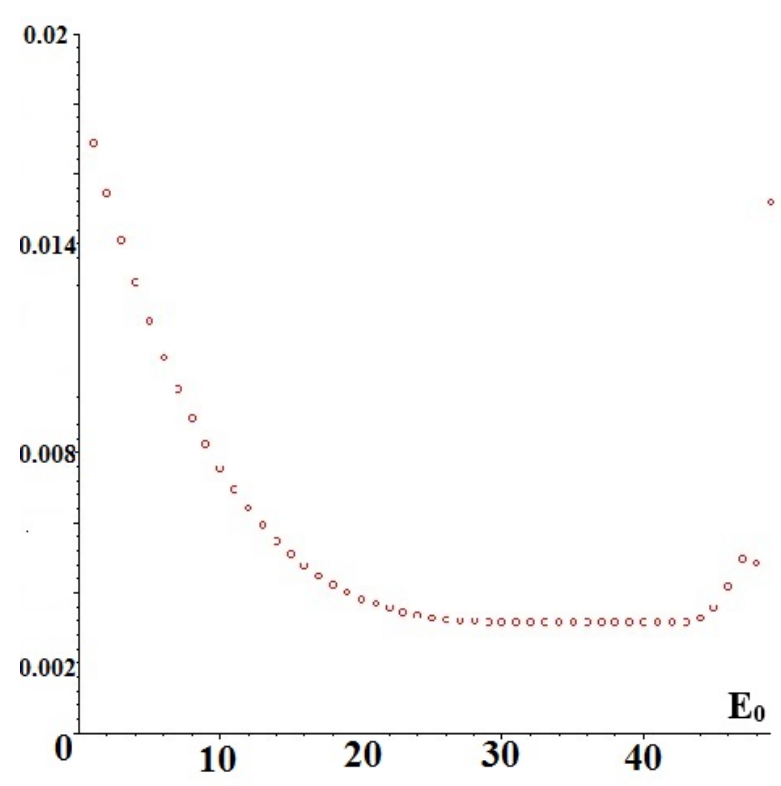

Figure 3. Quadratic discrepancy versus parameter $E_{0}$ for a group of first 4 energy levels of a sodium atom 
Let us describe an algorithm for restoring the effective potential parameters for a valence electron in an alkali metal atom and the parameters of the energy spectrum perturbation of this valence electron in the process of measurement.

1. Known "theoretical" spectrum (10) and (12) of the valence electron in an alkali metal atom, we can restore $\rho_{Z}=\sum f_{j}\left|\psi_{j}\right\rangle\left\langle\psi_{j}\right|$ from (12):

— for individual "segments" of the spectrum (10) and (12);

- for the "initial part" of the spectrum (10) and (12) from the spectrum ordered according to the Klechkovsky-Madelung rule.

2. Restore $V_{Z}(r)=\left(V_{1} * W_{\rho Z}\right)(r)$ from (11).

3. Compare the restored pseudopotential with the effective pseudopotential (9) (current verification).

4. Restore $\rho_{a p}=\sum c_{j}\left|\psi_{j}\right\rangle\left\langle\psi_{j}\right|$ from individual segments of table-defined spectrum using the calculated $\left\{f_{j}^{Z}\right\}$ from item 1:

$$
O_{\rho_{a p}}\left(H^{Z}\right)=O_{W}\left(H^{Z} * W_{\rho_{a p}}\right)=O_{W}\left(\left(H^{1} * W_{\rho_{Z}}\right) * W_{\rho_{a p}}\right) .
$$

5. Check by means of the calculated probabilities of "optical transitions" and compare with the data from [32] (final verification).

\section{Conclusion}

In Refs. [2], [28] the Kuryshkin-Wodkiewicz model of quantum measurement was implemented in application to the quantum Kepler problem. Earlier the quantum measurement model in certain modifications has been implemented in application to the quantum oscillator problem [3], [4]. In the present paper the Kuryshkin-Wodkiewicz model, realizing the Weyl-Kuryshkin quantization rule [2], [28], is generalized to quantum systems with one valence electron, e.g., atoms of alkali metals. The analysis is based on experimental data on the energy ordering of electrons in an atom according to the KlechkovskyMadelung rule and on the substantiation of a single-particle potential model for describing the energy spectrum of optical electrons in alkali metal atoms.

The author of Ref. [31] obtained a representation of the perturbation of a single-particle potential in the form of a convolution of the potential of an electron in a hydrogen atom with the Wigner function of a certain effective state of the core in an alkali metal atom. This representation allows reducing all calculation algorithms for alkali metals to the corresponding algorithms for the hydrogen atom. The proof of the model consistency is based on two Kato theorems [29], [30]. In the course of the proof, explicit formulas were obtained for the discrete spectrum of a valence electron for various spectral series, depending on the serial parameters of the disturbance of the spectrum of an isolated object in the process of quantum measurements.

\section{References}

[1] A. V. Zorin and L. A. Sevastianov, "Hydrogen-like atom with nonnegative quantum distribution function," Physics of Atomic Nuclei, no. 70, pp. 792-799, 2007. DOI: 10.1134/S1063778807040229. 
[2] L. Sevastyanov, A. Zorin, and A. Gorbachev, "Pseudo-Differential Operators in an Operational Model of the Quantum Measurement of Observables," in Mathematical Modeling and Computational Science, G. Adam, J. Buša, and M. Hnatič, Eds., vol. 7125, Berlin, Heidelberg: Springer Berlin Heidelberg, 2012, pp. 174-181. DOI: 10.1007/978-3642-28212-6_17.

[3] G. M. D'Ariano, U. Leonhardt, and H. Paul, "Homodyne detection of the density matrix of the radiation field," Phys. Rev. A, vol. 52, R1801R1804, 1995. DOI: 10.1103/PhysRevA.52.R1801.

[4] G. M. D'Ariano, "Measuring Quantum States," in Concepts and Advances in Quantum Optics and Spectroscopy of Solids, T. Hakioglu and A. S. Shumovsky, Eds., Amsterdam: Kluwer Acad. Publishers, 1997, pp. 175202.

[5] A. S. Holevo, Statistical Structure of Quantum Theory, ser. Lecture Notes in Physics Monographs. Berlin: Springer, 2001, vol. 67. DOI: 10.1007/3-540-44998-1.

[6] C. W. Helstrom, Quantum Detection and Estimation Theory. New York: Academic Press, 1976.

[7] G. Ludwig, Attempt of an axiomatic foundation of quantum mechanics and more general theories, II, ser. Commun. Math. Phys. 2001, vol. 4. DOI: $10.1007 / \mathrm{BF} 01653647$.

[8] E. B. Davies and J. T. Lewis, "An operational approach to quantum probability," Communications in Mathematical Physics, vol. 17, no. 3, pp. 239-260, 1970.

[9] M. Ozawa, Quantum reality and measurement: A quantum logical approach, ser. Foundations of Physics. 2011, vol. 41, pp. 592-607.

[10] M. Ozawa and Y. Kitajima, Reconstructing Bohr's Reply to EPR in Algebraic Quantum Theory, ser. Foundations of Physics. 2012, vol. 42, pp. 475-487. DOI: $10.1007 / \mathrm{s} 10701-011-9615-7$.

[11] A. V. Zorin, L. A. Sevastianov, and N. P. Tretyakov, "Computer modeling of hydrogen-like atoms in quantum mechanics with nonnegative distribution function," Programming and Computer Software, vol. 33, no. 2, pp. 94-104, 2007. DOI: 10.1134/S0361768807020077.

[12] V. A. Fock, Foundations of quantum mechanics. Mir Publishers, 1978.

[13] V. Kondratyev, The Structure of Atoms and Molecules. Univ Pr. of the Pacific, 2002.

[14] V. N. Ostrovsky, "What and How Physics Contributes to Understanding the Periodic Law," Foundations of Chemistry, no. 3, pp. 145-181, 2001. DOI: $10.1023 / \mathrm{A}: 1011476405933$.

[15] V. M. Klechkovskii, The Distribution of Atomic Electrons and the Rule of Successive Filling of $(n+l)$-Groups /Raspredelenie atomnyh elektronov i pravilo posledovatel'nogo zapolneniya $(n+l)$-grupp]. Moscow: Atomizdat, 1968, in Russian.

[16] E. Madelung, Die Mathematischen Hilfsmittel des Physikers, 3rd edition. Berlin: Springer, 1936. DOI: 10.1007/978-3-662-21800-6. 
[17] Y. N. Demkov and V. N. Ostrovskii, "Internal Symmetry of the Maxwell "Fish-eye" Problem and the Fock Group for the Hydrogen Atom," JETP, vol. 33, no. 6, pp. 1083-1087, 1971.

[18] Y. N. Demkov and V. N. Ostrovsky, " $n+1$ Filling Rule in the Periodic System and Focusing Potentials," JETP, vol. 35, no. 1, pp. 66-69, 1972.

[19] V. A. Fock, "Hydrogen atom and non-Euclidean geometry," Zs. Phys., vol. 98, p. 145, 1935.

[20] Y. Kitagawara and A. O. Barut, "Period doubling in the $\mathrm{n}+\mathrm{l}$ filling rule and dynamical symmetry of the Demkov-Ostrovsky atomic model," Journal of Physics B: Atomic and Molecular Physics, vol. 16, no. 18, pp. 3305-3327, 1983. DOI: 10.1088/0022-3700/16/18/006.

[21] Y. Kitagawara and A. O. Barut, "On the dynamical symmetry of the periodic table. II. Modified Demkov-Ostrovsky atomic model," Journal of Physics B: Atomic and Molecular Physics, vol. 17, no. 21, pp. 42514259, 1984. DOI: $10.1088 / 0022-3700 / 17 / 21 / 013$.

[22] A. L. Kholodenko. (2020). "From Mendeleev to Seiberg-Witten via Madelung. Available from." accessed Jul 16 2020, [Online]. Available: https://www.researchgate.net/publication/341597880.

[23] A. L. Kholodenko and L. H. Kauffman, "How the modified Bertrand theorem explains regularities of the periodic table I. From conformal invariance to Hopf mapping," 2019. arXiv: 1906.05278.

[24] Y. B. Rumer and A. I. Fet, "The group Spin (4) the Mendeleev system," Theor. Math. Phys, vol. 9, pp. 1081-1085, 1971. DOI: 10.1007/ BF01036944.

[25] V. V. Varlamov, "Group Theoretical Description of Periodic System of Elements [Teoretiko-gruppovoe opisanie periodicheskoj sistemy elementov]," Mathematical Structures and Modelling, vol. 46, no. 2, pp. 5-23, 2018, in Russian. DOI: 10.25513/2222-8772.2018.2.5-23.

[26] D. Kirzhnitz, Y. Lozovik, and G. Shpatkovskaya, "Statistical model of matter," Sov. Phys. Uspekhi., vol. 18, no. 9, pp. 649-672, 1975. DOI: 10.1070/PU1975v018n09ABEH005199.

[27] A. Fet, Group Theory of Chemical Elements. Berlin: de Gryuter, 2016.

[28] L. Sevastianov, A. Zorin, and A. Gorbachev, "A Quantum Measurements Model of Hydrogen-Like Atoms in Maple," in Computer Algebra in Scientific Computing, V. P. Gerdt, W. Koepf, E. W. Mayr, and E. V. Vorozhtsov, Eds., vol. 8136, Cham: Springer International Publishing, 2013, pp. 369-380. DOI: 10.1007/978-3-319-02297-0_30.

[29] B. Simon, "Tosio Kato's work on non-relativistic quantum mechanics: Part 1," Bulletin of Mathematical Sciences, no. 8, pp. 121-232, 2018. DoI: 10.1007/s13373-018-0118-0.

[30] B. Simon, "Tosio Kato's work on non-relativistic quantum mechanics, Part 2," Bulletin of Mathematical Sciences, vol. 9, no. 1, p. 1950005 , 2019. DOI: $10.1142 / \mathrm{S} 166436071950005 X$. 
[31] A. V. Zorin, "Approximate Computation of States with Minimal Dispersion in Kuryshkin-Wodkiewicz Quantum Mechanics," in 2019 11th International Congress on Ultra Modern Telecommunications and Control Systems and Workshops (ICUMT), Dublin, Ireland, 2019, 2019, pp. 1-5. DOI: $10.1109 /$ ICUMT48472.2019.8971007.

[32] (2020). "Physical reference data." accessed Jul 16 2020, [Online]. Available: https : / / www . nist . gov/pml/productsservices / physical reference-data/.

\section{For citation:}

A. V. Zorin, Kuryshkin-Wodkiewicz quantum measurement model for alkaline metal atoms, Discrete and Continuous Models and Applied Computational Science 28 (3) (2020) 274-288. DOI: 10.22363/2658-4670-2020-28-3-274-288.

\section{Information about the authors:}

Zorin, Alexander V. - Candidate of Physical and Mathematical Sciences, assistant professor of Department of Applied Probability and Informatics of Peoples' Friendship University of Russia (RUDN University) (e-mail: zorin-av@rudn.ru, phone: +7(495)9550927, ORCID: https://orcid.org/0000-0002-5721-4558, ResearcherID: AAH-4011-2019, Scopus Author ID: 57193219091) 
DOI: $10.22363 / 2658-4670-2020-28-3-274-288$

\title{
Модель квантовых измерений Курышкина-Вудкевича для атомов щелочных металлов
}

\author{
А. В. Зорин \\ Российский университет дружбы народов \\ ул. Миклухо-Маклая, д. 6, Москва, 117198, Россия
}

Конструктивная форма модели квантовых измерений Курышкина-Водкевича ранее была подробно разработана для квантовой задачи Кеплера. Для более сложных квантовых объектов такая конструкция неизвестна. В то же время стандартная (неконструктивная) модель квантовых измерений Холево-Хелстрома подходит для любого квантового объекта. В данной работе конструктивная модель квантовых измерений обобщена на более широкий класс квантовых объектов, то есть на оптический спектр атомов и ионов с одним валентным электроном. Анализ основан на экспериментальных данных об энергетическом упорядочении электронов в атоме по правилу Клечковского-Маделунга и на обосновании одночастичной потенциальной модели для описания энергетического спектра оптических электронов в атомах щелочных металлов. Представление возмущения одночастичного потенциала в виде свертки потенциала электрона в атоме водорода с функцией Вигнера некоторого эффективного состояния остова в представлении атома щелочного металла позволяет редуцировать все алгоритмы расчета для щелочных металлов к соответствующим алгоритмам для атома водорода.

Ключевые слова: модели квантовых измерений, энергетический спектр атомов щелочных металлов, метод одночастичного потенциала, модель квантовых измерений, возмущение дискретного спектра наблюдаемой 